\title{
ASYMPTOTIC BEHAVIOUR OF REPRODUCING KERNELS OF WEIGHTED BERGMAN SPACES
}

\author{
MIROSLAV ENGLIŠ
}

\begin{abstract}
Let $\Omega$ be a domain in $\mathbb{C}^{n}, F$ a nonnegative and $G$ a positive function on $\Omega$ such that $1 / G$ is locally bounded, $A_{\alpha}^{2}$ the space of all holomorphic functions on $\Omega$ square-integrable with respect to the measure $F^{\alpha} G d \lambda$, where $d \lambda$ is the $2 n$-dimensional Lebesgue measure, and $K_{\alpha}(x, y)$ the reproducing kernel for $A_{\alpha}^{2}$. It has been known for a long time that in some special situations (such as on bounded symmetric domains $\Omega$ with $G=\mathbf{1}$ and $F=$ the Bergman kernel function) the formula
\end{abstract}

$$
\lim _{\alpha \rightarrow+\infty} K_{\alpha}(x, x)^{1 / \alpha}=1 / F(x)
$$

holds true. [This fact even plays a crucial role in Berezin's theory of quantization on curved phase spaces.] In this paper we discuss the validity of this formula in the general case. The answer turns out to depend on, loosely speaking, how well the function $-\log F$ can be approximated by certain pluriharmonic functions lying below it. For instance, $(*)$ holds if $-\log F$ is convex (and, hence, can be approximated from below by linear functions), for any function $G$. Counterexamples are also given to show that in general $(*)$ may fail drastically, or even be true for some $x$ and fail for the remaining ones. Finally, we also consider the question of convergence of $K_{\alpha}(x, y)^{1 / \alpha}$ for $x \neq y$, which leads to an unexpected result showing that the zeroes of the reproducing kernels are affected by the smoothness of $F$ : for instance, if $F$ is not real-analytic at some point, then $K_{\alpha}(x, y)$ must have zeroes for all $\alpha$ sufficiently large.

\section{INTRODUCTION AND RESULTS}

Let $\Omega$ be a domain in $\mathbb{C}^{n}$ and $F, G$ nonnegative measurable functions on $\Omega$ such that $G>0$ and $1 / G$ is locally bounded. The weighted Bergman space appearing in the title is

$$
A_{\alpha}^{2}=\left\{f \text { holomorphic on } \Omega:\left(\int_{\Omega}|f|^{2} F^{\alpha} G d \lambda\right)^{1 / 2} \equiv\|f\|_{\alpha}<+\infty\right\} .
$$

Here $d \lambda$ stands for the Lebesgue measure and $\alpha$ is a real number.

The reproducing kernel for $A_{\alpha}^{2}$ is the function $K_{\alpha}(x, y)$ of two variables $x, y \in \Omega$, holomorphic in $x$ and anti-holomorphic in $y$, such that $K_{\alpha}(\cdot, y) \in A_{\alpha}^{2}$ for each $y$ and

$$
f(y)=\int_{\Omega} f(x) \overline{K_{\alpha}(x, y)} F(x)^{\alpha} G(x) d \lambda(x) \quad \forall y \in \Omega \forall f \in A_{\alpha}^{2} .
$$

Received by the editors March 22, 1996.

1991 Mathematics Subject Classification. Primary 30C40, 32H10; Secondary 31C10, 30E15.

Key words and phrases. Bergman space, reproducing kernels, asymptotic behaviour, lower pluriharmonic envelopes, plurisubharmonic functions.

The author's research was supported by GA AV ČR grants C1019601 and 119106 and by GA ČR grant 201/96/0411.

(C)1997 American Mathematical Society 
Under suitable hypothesis on $F$ (for instance, when $F>0$ and $1 / F$ is locally bounded) and the stated hypothesis on $G$, it is known that the reproducing kernel exists and is unique $([6],[15])$ and the value $K_{\alpha}(x, x)$ coincides with the square $e_{\alpha}(x)$ of the norm of the evaluation functional at $x$ on $A_{\alpha}^{2}$ :

$$
K_{\alpha}(x, x)=e_{\alpha}(x) \equiv \sup \left\{|f(x)|^{2} ; f \in A_{\alpha}^{2},\|f\|_{\alpha} \leq 1\right\} .
$$

Our main concern here will be the limit

$$
\rho(x) \equiv \lim _{\alpha \rightarrow+\infty} K_{\alpha}(x, x)^{1 / \alpha}=\lim _{\alpha \rightarrow+\infty} e_{\alpha}(x)^{1 / \alpha} .
$$

There is a priori no reason for this limit even to exist. However, in many important situations the limit does exist, and, moreover, is equal to

$$
\rho(x)=\frac{1}{F(x)} \text {. }
$$

Instances of this situation include the following:

1. $\Omega=\mathbb{D}$, the unit disc in $\mathbb{C} ; G=\mathbf{1}$ (the constant one), $F(z)=1-|z|^{2}$. (Folklore; known, at least, already to Poincaré.)

2. The Segal-Bargmann (or Fock) spaces: $\Omega=\mathbb{C}^{n}, G=\mathbf{1}, F=e^{-|z|^{2}}$ ([4], [5], [17], [1]).

3. (a generalization of 1.) $\Omega=$ the unit ball of $\mathbb{C}^{n}, G=\mathbf{1}, F=1-\|z\|^{2} \quad$ [22].

4. $\Omega=$ a bounded symmetric domain, $G=\mathbf{1}, F=$ the Bergman kernel function $([3],[16],[20],[12],[13])$.

5. $\Omega$ a domain in $\mathbb{C}$ of hyperbolic type, $G=\mathbf{1}, F(\phi(z))=\left(1-|z|^{2}\right) \cdot\left|\phi^{\prime}(z)\right|$ where $\phi: \mathbb{D} \rightarrow \Omega$ is any uniformization map (that is, $d s^{2}=F(z)|d z|^{2}$ is the Poincaré metric on $\Omega)([19]$, [9]).

6. Some pseudoconvex domains in $\mathbb{C}^{2}$ equipped with a Kähler metric $g_{i \bar{j}} d z_{i} d \bar{z}_{j}$, with $g_{i \bar{j}}=\left(\partial^{2} \Psi / \partial z_{i} \partial \bar{z}_{j}\right), F=e^{-\Psi}, G=\operatorname{det}\left(g_{i \bar{j}}\right)$, where $\Psi$ is a real-valued strictly plurisubharmonic function (the Kähler potential) [11].

In this note we will show that the following general result holds.

Theorem A. Let $F \geq 0$ and $G>0$ be measurable functions on $\Omega$ such that $1 / G$ is locally bounded. Suppose that $-\log F$ is a convex function and that $\mathbf{1} \in A_{\alpha}^{2}$ for some $\alpha>0$. Then the limit

$$
\lim _{\alpha \rightarrow+\infty} e_{\alpha}(x)^{1 / \alpha} \equiv \rho(x)
$$

exists and is equal to $1 / F(x)$.

The limits above are of central importance in some approaches to quantization on $\Omega$. See [3], [10], [11], [8], [26], [25].

A more detailed description involves the auxiliary functions $F^{*}, F^{* *}, F^{* * *}, F^{\#}$ defined by

$$
\begin{aligned}
1 / F^{*} & =\sup \left\{\left|e^{g}\right|^{2}: g \text { holomorphic on } \Omega,\left|e^{g}\right|^{2} \leq 1 / F, e^{\alpha g} \in A_{\alpha}^{2} \text { for some } \alpha>0\right\}, \\
1 / F^{* *} & =\sup \left\{\left|e^{g}\right|^{2}: g \text { holomorphic on } \Omega,\left|e^{g}\right|^{2} \leq 1 / F\right\}, \\
1 / F^{* * *} & =\sup \left\{|f|^{\varkappa}: f \text { holomorphic on } \Omega, \varkappa>0,|f|^{\varkappa} \leq 1 / F\right\}, \\
1 / F^{\#} & =\sup \left\{e^{\psi}: \psi \text { is plurisubharmonic and } e^{\psi(x)} \leq 1 / \lim _{\epsilon \backslash 0} \inf _{|x-y|<\epsilon} F(y)\right\} .
\end{aligned}
$$

(In other words, for $F$ lower semicontinuous, $-\log F^{\#}$ is the greatest plurisubharmonic function majorized by $-\log F$.) The condition that $e^{\alpha g} \in A_{\alpha}^{2}$ for some $\alpha>0$ 
can be equivalently stated as $\left|e^{g}\right|^{2} F \in L^{\alpha}(\Omega, G d \lambda)$ for some $\alpha>0$; if the measure $G d \lambda$ is finite, this condition can even be omitted completely, and $F^{*}=F^{* *}$. In general, we only have $F^{*} \geq F^{* *} \geq F^{* * *} \geq F$ and $F^{* * *} \geq F^{\#} \geq F_{\text {lsc }}$, where $F_{\text {lsc }}$ denotes the lower-semicontinuous regularization of $F$.

Theorem B. Let $F \geq 0$ and $G>0$ be measurable functions on $\Omega$ such that $1 / G$ is locally bounded. Then

$$
1 / F^{\#}(x) \geq \limsup _{\alpha \rightarrow+\infty} e_{\alpha}(x)^{1 / \alpha} \geq \liminf _{\alpha \rightarrow+\infty} e_{\alpha}(x)^{1 / \alpha} \geq 1 / F^{*}(x) .
$$

If $\mathbf{1} \in A_{\alpha}^{2}$ for some $\alpha>0$, then even

$$
\liminf _{\alpha \rightarrow+\infty} e_{\alpha}(x)^{1 / \alpha} \geq 1 / F^{* *}(x) .
$$

We also have a sharper lower bound for $\lim \sup _{\alpha \rightarrow+\infty} e_{\alpha}^{1 / \alpha}$ :

Theorem $\mathbf{B}^{\prime}$. Let $F \geq 0$ and $G>0$ be measurable functions on $\Omega$ such that $1 / G$ is locally bounded and $\mathbf{1} \in A_{\alpha_{0}}^{2}$ for some $\alpha_{0}>0$. Then

$$
\limsup _{\alpha \rightarrow+\infty} e_{\alpha}(x)^{1 / \alpha} \geq 1 / F^{* * *}(x) .
$$

If $F$ is also positive and $1 / F$ locally bounded (so that the reproducing kernels $K_{\alpha}(x, y)$ - not only $e_{\alpha}(x)$ - are defined), we can also consider the convergence of $K_{\alpha}(x, y)^{1 / \alpha}$ on $\Omega \times \Omega$, provided the $\alpha$-th root makes sense. This will reveal the following surprising connection between the zeroes of $K_{\alpha}(x, y)$ and the smoothness of $F$.

Theorem C. Assume that $F, G>0,1 / F$ and $1 / G$ are locally bounded and the limit $\rho(x)$ exists and equals $1 / F$. Suppose further that there exist an unbounded subset $A$ of $[1,+\infty)$ and a simply-connected open set $U \subset \Omega$ such that

$$
K_{\alpha}(x, y) \neq 0 \quad \text { for all } \alpha \in A \text { and } x, y \in U \text {. }
$$

Then $F(x)$ extends to a zero-free function $F(x, y)$ on $U \times U$, holomorphic in $x$ and anti-holomorphic in $y$, such that $F(x, x)=F(x)$ and $|F(x, y)|^{2} \geq F(x, x) F(y, y)$.

Corollary. Assume that $F, G>0,1 / F$ and $1 / G$ are locally bounded and the limit $\rho(x)$ exists and equals $1 / F$. Suppose further that $F$ is not real analytic at some point $z_{0} \in \Omega$. Then for any sequence $\alpha_{k} \rightarrow \infty$ there exist a subsequence $\alpha_{k_{j}}$ and points $x_{j}, y_{j} \in \Omega$ such that both $\left\{x_{j}\right\}$ and $\left\{y_{j}\right\}$ converge to $z_{0}$ and $K_{\alpha_{k_{j}}}\left(x_{j}, y_{j}\right)=0$ for each $j$. (In other words, the point $\left(z_{0}, z_{0}\right)$ is an accumulation point of zeroes of the functions $K_{\alpha}(x, y)$.)

Theorems A, B and B' are proved in Section 2, Theorem C in Section 3. Section 4 brings some examples, and the last Section 5 mentions briefly some open problems.

Throughout the text, the letters $d \mu$ stand for the measure $d \mu(x)=G(x) d \lambda(x)$, and $d \mu_{\alpha}$ denotes the measure $d \mu_{\alpha}=F^{\alpha} d \mu$. If a function $u$ taking values in the interval $[-\infty,+\infty)$ is locally bounded from above, we will denote by $u_{\text {usc }}$ its uppersemicontinuous regularization

$$
u_{\mathrm{usc}}(x):=\lim _{\epsilon \searrow 0} \sup _{y \in D(x, \epsilon)} u(y) .
$$


Clearly $u \leq u_{\mathrm{usc}}$, and equality prevails iff $u$ is upper semicontinuous. The lowersemicontinuous regularization can be defined analogously. The functions $\bar{\rho}(x)$ and $\rho(x)$ are abbreviations for

$$
\bar{\rho}(x):=\limsup _{\alpha \rightarrow+\infty} e_{\alpha}(x)^{1 / \alpha} \quad \text { and } \quad \underline{\rho}(x):=\liminf _{\alpha \rightarrow+\infty} e_{\alpha}(x)^{1 / \alpha},
$$

respectively; and PSH stands for "plurisubharmonic".

\section{The Limit of $e_{\alpha}(x)^{1 / \alpha}$}

Proof of Theorem $B$. Let $r>0$ be such that the closed polydisc $\bar{D}=\overline{D(x, r)}$ lies wholly in $\Omega$. By the mean value theorem for holomorphic functions,

$$
f(x)=\left(\pi r^{2}\right)^{-n} \int_{D} f d \lambda=\left(\pi r^{2}\right)^{-n} \int_{D} \frac{f}{F^{\alpha} G} d \mu_{\alpha}
$$

for any holomorphic function $f$ on $\Omega$. By the Schwarz inequality,

$$
\begin{aligned}
|f(x)|^{2} & \leq\|f\|_{\alpha}^{2} \cdot\left(\int_{D}\left(\pi r^{2}\right)^{-2 n} F^{-2 \alpha} G^{-2} d \mu_{\alpha}\right) \\
& =\|f\|_{\alpha}^{2} \cdot\left(\pi r^{2}\right)^{-2 n} \int_{D} F^{-\alpha} G^{-1} d \lambda .
\end{aligned}
$$

It follows that

$$
\begin{aligned}
e_{\alpha}(x) & \leq\left(\pi r^{2}\right)^{-2 n} \int_{D} F^{-\alpha} G^{-1} d \lambda \\
& \leq\left(\pi r^{2}\right)^{-n} \cdot \sup _{D} \frac{1}{G} \cdot\left(\inf _{D} F\right)^{-\alpha} .
\end{aligned}
$$

Taking roots gives

$$
e_{\alpha}(x)^{1 / \alpha} \leq\left(\frac{\sup _{D} 1 / G}{\pi^{n} r^{2 n}}\right)^{1 / \alpha} \cdot\left(\inf _{D} F\right)^{-1} .
$$

Note that the last supremum is finite by hypothesis. Letting $\alpha$ tend to infinity, we therefore obtain

$$
\bar{\rho}(x) \leq 1 / \inf _{D(x, r)} F .
$$

This holds for all sufficiently small positive $r$. Letting $r \rightarrow 0$ yields

$$
\bar{\rho}(x) \leq 1 / \lim _{r \searrow 0} \inf _{D(x, r)} F=1 / F_{\mathrm{lsc}}(x) .
$$

On the other hand, by the definition of $e_{\alpha}$,

$$
e_{\alpha}(x)^{1 / \alpha}=\sup \left\{|f(x)|^{2 / \alpha}: f \text { holomorphic on } \Omega,\|f\|_{\alpha} \leq 1\right\} .
$$

Consequently,

$$
\log e_{\alpha}(x)^{1 / \alpha}=\sup \left\{\frac{2}{\alpha} \log |f(x)|: f \text { holomorphic on } \Omega,\|f\|_{\alpha} \leq 1\right\},
$$

and

(3) $\log \bar{\rho}(x)=\lim _{k \rightarrow+\infty} \sup \left\{\frac{2}{\alpha} \log |f(x)|: \alpha \geq k,\|f\|_{\alpha} \leq 1, f\right.$ holomorphic on $\left.\Omega\right\}$.

Recall now the following well-known facts from the theory of plurisubharmonic functions:

(a) If $u_{k}$ is a decreasing sequence of PSH functions, then $u=\lim _{k \rightarrow \infty} u_{k}$ is also plurisubharmonic. 
(b) If $\left\{u_{\iota}\right\}_{\iota \in I}$ is a family of PSH functions such that its supremum $u=\sup _{\iota \in I} u_{\iota}$ is locally bounded from above, then the upper-semicontinuous regularization $u_{\text {usc }}$ of $u$ is also plurisubharmonic.

(c) Let $u$ be a function locally bounded from above and $u_{r}(x):=\sup _{D(x, r)} u$ (so $\left.u_{\text {usc }}=\lim _{r \searrow 0} u_{r}\right)$. Then $\lim _{r \searrow 0}\left(u_{r}\right)_{\text {usc }}=u_{\text {usc }}$.

[For proofs of (a) and (b) see e.g. [18], Theorem 2.9.14. For (c), observe first that $\left(u_{r}\right)_{\mathrm{usc}} \leq u_{(1+\delta) r}$ for any $\delta>0$, by the triangle inequality; combining this with the trivial fact that $\left(u_{r}\right)_{\mathrm{usc}} \geq u_{r}$ and letting $r$ tend to zero gives the result.]

For brevity, let us temporarily denote

$$
\begin{aligned}
U_{k}(x) & :=\sup \left\{\frac{2}{\alpha} \log |f(x)|: \alpha \geq k,\|f\|_{\alpha} \leq 1, f \text { holomorphic on } \Omega\right\} \\
& =\sup _{\alpha \geq k} \log e_{\alpha}(x)^{1 / \alpha}, \\
C_{r}(x) & :=\max \left[1, \frac{\sup _{D(x, r)} 1 / G}{\pi^{n} r^{2 n}}\right] .
\end{aligned}
$$

Then $U_{k} \searrow \log \bar{\rho}$ as $k \rightarrow \infty$ and, in view of (2),

$$
U_{k}(x) \leq \sup _{\alpha \geq k} \log \frac{C_{r}(x)^{1 / \alpha}}{\inf _{D(x, r)} F}=\log \frac{C_{r}(x)^{1 / k}}{\inf _{D(x, r)} F},
$$

so the functions $U_{k}$ are locally bounded from above. Moreover,

$$
\begin{aligned}
\lim _{k \rightarrow \infty}\left(U_{k}\right)_{\mathrm{usc}} & \leq \lim _{k \rightarrow \infty}\left[\frac{1}{k}\left(\log C_{r}\right)_{\mathrm{usc}}+\left(-\log \inf _{D(x, r)} F\right)_{\mathrm{usc}}\right] \\
& =\left(\log \sup _{D(x, r)} 1 / F\right)_{\mathrm{usc} .}
\end{aligned}
$$

The left-hand side is independent of $r$; letting $r \rightarrow 0$ yields, by (c) above,

$$
\lim _{k \rightarrow \infty}\left(U_{k}\right)_{\mathrm{usc}} \leq(-\log F)_{\mathrm{usc}} .
$$

In view of (b), and since $\log |f|$ is plurisubharmonic for any holomorphic function $f$, each $\left(U_{k}\right)_{\text {usc }}$ is a PSH function. The sequence $U_{k}$ being decreasing, (a) implies that $\lim _{k \rightarrow \infty}\left(U_{k}\right)_{\text {usc }}$ is also plurisubharmonic. Since the greatest PSH function majorized by $(-\log F)_{\mathrm{usc}}$ is $-\log F^{\#}$ by definition, we see that

$$
\lim _{k \rightarrow \infty}\left(U_{k}\right)_{\mathrm{usc}} \leq-\log F^{\#} \text {. }
$$

As $u \leq u_{\text {usc }}$ for any function $u$ and $U_{k} \searrow \log \bar{\rho}$, we therefore have

$$
\log \bar{\rho}=\lim _{k \rightarrow \infty} U_{k} \leq \lim _{k \rightarrow \infty}\left(U_{k}\right)_{\mathrm{usc}} \leq-\log F^{\#},
$$

and the first half of Theorem B follows.

To prove the other half, consider an arbitrary holomorphic function $f$ on $\Omega$ which does not vanish identically. Then

$$
e_{\alpha}(x) \geq \frac{|f(x)|^{2}}{\|f\|_{\alpha}^{2}} .
$$

Indeed, for $f \in A_{\alpha}^{2}$, this is just the definition of $e_{\alpha}(x)$, and for $f \notin A_{\alpha}^{2}$, the righthand side is zero by the usual convention $1 /+\infty=0$. Taking in particular $f=e^{\alpha g}$, 
we see that

$$
e_{\alpha}(x) \geq \frac{\left|e^{\alpha g(x)}\right|^{2}}{\left\|e^{\alpha g}\right\|_{\alpha}^{2}}=\frac{\left|e^{g(x)}\right|^{2 \alpha}}{\int\left(\left|e^{g}\right|^{2} F\right)^{\alpha} d \mu}
$$

for any holomorphic function $g$. Thus

$$
e_{\alpha}(x)^{1 / \alpha} \geq \frac{\left|e^{g(x)}\right|^{2}}{\left\|\left|e^{g}\right|^{2} F\right\|_{L^{\alpha}(d \mu)}} .
$$

Taking the limit gives

$$
\underline{\rho}(x) \geq \frac{\left|e^{g(x)}\right|^{2}}{\left\|\left|e^{g}\right|^{2} F\right\|_{*, d \mu}}
$$

where $\|\cdot\|_{*, d \mu}$ is defined as

$\|\phi\|_{*, d \mu} \equiv \lim _{p \rightarrow \infty}\|\phi\|_{L^{p}(d \mu)}=\left\{\begin{array}{l}\|\phi\|_{\infty} \text { if } \phi \in L^{p}(d \mu) \forall p \in\left(p_{0}, \infty\right) \text { for some finite } p_{0}, \\ +\infty \text { otherwise. }\end{array}\right.$

Assume further that $\left|e^{g}\right|^{2} \leq 1 / F$ and $e^{\alpha g} \in A_{\alpha}^{2}$ for some $\alpha>0$. In other words, $\left|e^{g}\right|^{2} F \in L^{\infty}(d \mu) \cap L^{\alpha}(d \mu)$; thus, since $\log \|\cdot\|_{p}$ is a convex function of $\frac{1}{p}$, we have $\left|e^{g}\right|^{2} F \in L^{p}(d \mu) \forall p \in[\alpha, \infty]$, and $\left\|\left|e^{g}\right|^{2} F\right\|_{*, d \mu}=\left\|\left|e^{g}\right|^{2} F\right\|_{\infty}$. Consequently,

$$
\underline{\rho}(x) \geq \frac{\left|e^{g(x)}\right|^{2}}{\left\|\left|e^{g}\right|^{2} F\right\|_{\infty}} \geq\left|e^{g(x)}\right|^{2} .
$$

Summing up, we see that

$\underline{\rho}(x) \geq \sup \left\{\left|e^{g(x)}\right|^{2}: g\right.$ holomorphic on $\Omega,\left|e^{g}\right|^{2} \leq 1 / F, e^{\alpha g} \in A_{\alpha}^{2}$ for some $\left.\alpha>0\right\}$, or $\rho(x) \geq 1 / F^{*}(x)$, as asserted.

If $1 \in A_{\alpha_{0}}^{2}$ for some $\alpha_{0}$, we instead take $f=e^{\left(\alpha-\alpha_{0}\right) g}$ in (4). Proceeding as above, we see that

$$
e_{\alpha}(x)^{1 / \alpha} \geq \frac{\left|e^{\left(\alpha-\alpha_{0}\right) g(x)}\right|^{2 / \alpha}}{\left\|\left|e^{g}\right|^{2} F\right\|_{L^{\alpha-\alpha_{0}}\left(d \mu_{\alpha_{0}}\right)}}
$$

and

$$
\underline{\rho}(x) \geq \frac{\left|e^{g(x)}\right|^{2}}{\left\|\left|e^{g}\right|^{2} F\right\|_{*, d \mu_{\alpha_{0}}}} .
$$

Assume that $\left|e^{g}\right|^{2} \leq 1 / F$. Then $\left|e^{g}\right|^{2} F \in L^{\infty}\left(d \mu_{\alpha_{0}}\right)$; owing to the finiteness of $d \mu_{\alpha_{0}}$, this implies $\left|e^{g}\right|^{2} F \in L^{p}\left(d \mu_{\alpha_{0}}\right) \forall p>0$. Thus again $\|\cdot\|_{*, d \mu_{\alpha_{0}}}=\|\cdot\|_{\infty}$, and

$$
\underline{\rho}(x) \geq \frac{\left|e^{g(x)}\right|^{2}}{\left\|\left|e^{g}\right|^{2} F\right\|_{\infty}} \geq\left|e^{g(x)}\right|^{2},
$$

so

$$
\underline{\rho}(x) \geq \sup \left\{\left|e^{g(x)}\right|^{2}: g \text { holomorphic on } \Omega,\left|e^{g}\right|^{2} \leq 1 / F\right\}=1 / F^{* *},
$$

which is what we wanted to prove. 
Proof of Theorem A. Any convex function on $\mathbb{R}^{2 n}$ is the supremum of the affine functions lying below it. (An affine function is a sum of a real-linear function and a constant.) Thus, if $-\log F$ is convex, we have

$$
\begin{aligned}
-\log F & =\sup \{\phi: \quad \phi \text { affine, } \phi \leq-\log F\} \\
& =\sup \left\{2 \operatorname{Re} g: g(z)=\langle z, c\rangle+d\left(c \in \mathbb{C}^{n}, d \in \mathbb{C}\right), 2 \operatorname{Re} g \leq-\log F\right\} .
\end{aligned}
$$

Therefore

$$
\begin{aligned}
1 / F & =\sup \left\{\left|e^{g}\right|^{2}: g(z)=\langle z, c\rangle+d\left(c \in \mathbb{C}^{n}, d \in \mathbb{C}\right),\left|e^{g}\right|^{2} \leq 1 / F\right\} \\
& \leq \sup \left\{\left|e^{g}\right|^{2}: g \text { holomorphic on } \Omega,\left|e^{g}\right|^{2} \leq 1 / F\right\}=1 / F^{* *},
\end{aligned}
$$

so $F^{* *}=F$, and an application of Theorem $\mathrm{B}$ completes the proof.

Remark. The assumption that $\alpha>0$ in Theorem A can in fact be relaxed to $\alpha \geq 0$. If $\mathbf{1} \in A_{0}^{2}$, i.e. if the measure $\mu$ is finite, then - as was already noted in the Introduction - we have $F^{*}=F^{* *}$. On the other hand, the preceding paragraph shows that $F^{* *}=F$. It only remains to apply Theorem B.

Proof of Theorem $\mathrm{B}^{\prime}$. Let $f$ be a holomorphic function on $\Omega$, not identically zero, such that $|f|^{2 / \gamma} \leq 1 / F$ for some $\gamma>0$. Let us take in (4) $f=f^{k}$ and $\alpha=\alpha_{0}+k \gamma$, where $k$ is an arbitrary positive integer. We obtain

$$
e_{\alpha_{0}+k \gamma}(x) \geq \frac{\left|f^{k}(x)\right|^{2}}{\int_{\Omega}|f|^{2 k} F^{k \gamma} d \mu_{\alpha_{0}}} .
$$

Therefore

$$
e_{\alpha_{0}+k \gamma}(x)^{1 / k \gamma} \geq \frac{|f(x)|^{2 / \gamma}}{\left\||f|^{2 / \gamma} F\right\|_{L^{k \gamma}\left(d \mu_{\alpha_{0}}\right)}} .
$$

Passing to the limit superior as $k \rightarrow \infty$, we get

$$
\bar{\rho}(x) \geq \limsup _{k \rightarrow \infty} e_{\alpha_{0}+k \gamma}(x)^{1 / k \gamma} \geq \frac{|f(x)|^{2 / \gamma}}{\left\||f|^{2 / \gamma} F\right\|_{*, d \mu_{\alpha_{0}}}} .
$$

Again, the finiteness of $d \mu_{\alpha_{0}}$ implies that

$$
\left\||f|^{2 / \gamma} F\right\|_{*, d \mu_{\alpha_{0}}}=\left\||f|^{2 / \gamma} F\right\|_{\infty} \leq 1,
$$

and we conclude that

$$
\bar{\rho}(x) \geq|f(x)|^{2 / \gamma}
$$

for all holomorphic functions $f$ and $\gamma>0$ such that $|f|^{2 / \gamma} \leq 1 / F$. By definition (replacing $2 / \gamma$ by $\varkappa$ ), this means that $\bar{\rho} \geq 1 / F^{* * *}$, which completes the proof.

It would be of interest to know in general for which functions $F$ and $G$ one has $F^{* *}=F$, or $F^{*}=F$. A closely related question is that of characterizing the functions $\phi$ of the form

$$
\phi=\sup \{\psi: \psi \leq \phi, \psi \text { harmonic }\},
$$

i.e. the suprema of harmonic functions; the class of all functions of this form which are locally bounded from above is sometimes denoted $\mathcal{H}^{\sup }(\Omega)$ in the literature. Clearly on a simply connected planar domain, $F=F^{* *}$ is equivalent to $-\log F \in \mathcal{H}^{\sup }(\Omega)$. Also, any upper semicontinuous function in $\mathcal{H}^{\text {sup }}$ is necessarily plurisubharmonic, since the upper-semicontinuous regularization of a supremum of 
pluri(sub)harmonic functions is again a plurisubharmonic function. The converse is false: if $\phi$ is defined on the unit disc as

$$
\phi(z)=\max (A, \log |z|)
$$

for some constant $A<0$, then $\phi$ is subharmonic and any harmonic function $\psi \leq \phi$ must satisfy

$$
\psi(z) \leq A \frac{1-|z|}{1+|z|}
$$

by the Harnack inequality; however, the right-hand side is $<\phi(z)$ as soon as $|A|$ is sufficiently large. (The author is indebted to Ivan Netuka [21] for this counterexample.) The class $\mathcal{H}^{\text {sup }}$ has recently been studied by Vondracek [27], [28].

More generally, let $\mathcal{H}(\Omega)$ and $P S H(\Omega)$ stand for harmonic and plurisubharmonic functions on $\Omega$, respectively; denote

$$
\begin{aligned}
\mathcal{H}_{0}(\Omega) & =\{\operatorname{Re} f: f \text { holomorphic on } \Omega\}, \\
\mathcal{G}(\Omega) & =\{\varkappa \log |f|: f \text { holomorphic on } \Omega, \varkappa>0\}, \\
L B A(\Omega) & =\text { functions on } \Omega \text { which are locally bounded from above, }
\end{aligned}
$$

and, in addition to

$$
\mathcal{H}^{\text {sup }}(\Omega)=\left\{\phi \in L B A: \phi=\sup _{\alpha} \psi_{\alpha}, \psi_{\alpha} \in \mathcal{H}\right\}
$$

(the suprema of harmonic functions) defined above, introduce the function classes $\mathcal{H}_{0}^{\text {sup }}=\left\{\phi \in L B A: \phi=\sup _{\alpha} \psi_{\alpha}, \psi_{\alpha} \in \mathcal{H}_{0}\right\} \quad$ (suprema of functions from $\mathcal{H}_{0}$ ), $\mathcal{G}^{\text {sup }}=\left\{\phi \in L B A: \phi=\sup _{\alpha} \psi_{\alpha}, \psi_{\alpha} \in \mathcal{G}\right\} \quad$ (suprema of functions from $\mathcal{G}$ ), $\mathcal{G}^{\text {sup }}=\left\{\phi: \exists \psi_{n} \in \mathcal{G}^{\text {sup }}, \psi_{n} \searrow \phi\right\} \quad$ (decreasing limits of function from $\mathcal{G}^{\text {sup }}$ ), and for a function $\phi$ locally bounded from above on $\Omega$, define

$$
\begin{aligned}
\phi_{0}^{\mathcal{H}} & =\sup \left\{\psi: \psi \in \mathcal{H}_{0}, \psi \leq \phi\right\} \quad\left(=\sup \left\{\psi: \psi \in \mathcal{H}_{0}^{\text {sup }}, \psi \leq \phi\right\}\right), \\
\phi^{\mathcal{G}} & =\sup \{\psi: \psi \in \mathcal{G}, \psi \leq \phi\} \quad\left(=\sup \left\{\psi: \psi \in \mathcal{G}^{\text {sup }}, \psi \leq \phi\right\}\right), \\
\phi^{\mathcal{G}} & =\sup \left\{\psi: \psi \in \mathcal{G}_{\searrow}^{\text {sup }}, \psi \leq \phi_{\text {usc }}\right\}, \\
\phi^{P S H} & =\sup \left\{\psi: \psi \in P S H, \psi \leq \phi_{\text {usc }}\right\} .
\end{aligned}
$$

In particular, for $\phi=-\log F$ these definitions turn into

$$
\phi_{0}^{\mathcal{H}}=-\log F^{* *}, \quad \phi^{\mathcal{G}}=-\log F^{* * *}, \quad \phi^{P S H}=-\log F^{\#} .
$$

Clearly we have the containments

$$
\mathcal{H}_{0}^{\text {sup }} \varsubsetneqq \mathcal{G}^{\text {sup }} \varsubsetneqq \mathcal{G}^{\text {sup }}
$$

The first inclusion is immediate, and is strict because of the example in the preceding paragraph. The second inclusion is strict because for $\Omega=\mathbb{D} \backslash\{0\}$, the function

$$
\phi(x)=\sum_{j=2}^{\infty} 2^{-j} \log \left|\frac{x-1 / j}{1-x / j}\right|^{2}
$$

belongs to $\mathcal{G}_{\searrow}^{\text {sup }}$ (the partial sums of the series on the right-hand side belong to $\mathcal{G}$ and decrease to $\phi$ ), yet any holomorphic function on $\mathbb{D} \backslash\{0\}$ satisfying $|f|^{\varkappa} \leq e^{\phi}$ with $\varkappa>0$ is bounded (by 1 ) and vanishes at $x=\frac{1}{j}(j=2,3, \ldots)$, hence must be identically 0 by Riemann's Removable Singularities Theorem. 
Gathering up the information from our theorems and combining it with (7), we see that our findings so far can be summarized as

$$
\phi_{0}^{\mathcal{H}} \leq \phi^{\mathcal{G}} \leq \log \bar{\rho} \leq \phi^{\mathcal{G}}
$$

and

$$
\phi_{0}^{\mathcal{H}} \leq \log \underline{\rho} \leq \log \bar{\rho} \leq \phi^{P S H} \leq \phi_{\text {usc }}
$$

where $\phi:=-\log F$ and we assume that $1 \in A_{\alpha_{0}}^{2}$ for some $\alpha_{0}>0$. Here the third inequality in (9) is a consequence of $\log \bar{\rho} \in \mathcal{G}^{\text {sup }}$, which in turn follows from (3); the second inequality is the content of Theorem $\mathrm{B}^{\prime}$. Also, as observed above,

$$
\begin{gathered}
F=F^{* *} \Longleftrightarrow \phi \in \mathcal{H}_{0}^{\text {sup }} \Longleftrightarrow \phi=\phi_{0}^{\mathcal{H}}, \\
F=F^{* * *} \Longleftrightarrow \phi \in \mathcal{G}^{\text {sup }} \Longleftrightarrow \phi=\phi^{\mathcal{G}}, \\
F=F^{\#} \Longleftrightarrow \phi \in P S H \Longleftrightarrow \phi=\phi^{P S H},
\end{gathered}
$$

etc. It would be particularly interesting to know when one has $\phi^{\mathcal{G}}=\phi^{\mathcal{G}}$, or at least $\phi^{\mathcal{G}}=\phi^{P S H}$. Note that, even though no investigations of the specific situation encountered here are known to the author, the study of various "envelopes" of the form (6) is a standard topic in the literature, in particular in the context of abstract (=Choquet, Shilov, etc.) boundaries; see e.g. the excellent paper on Korovkin theorems by Bauer [2].

\section{The Limit of $K_{\alpha}(x, y)^{1 / \alpha}$}

Proof of Theorem C. By the reproducing property of $K_{\alpha}$ and the Schwarz inequality, we have

$$
\left|K_{\alpha}(x, y)\right|^{2} \leq K_{\alpha}(x, x) \cdot K_{\alpha}(y, y) \equiv e_{\alpha}(x) e_{\alpha}(y),
$$

so

$$
\left|K_{\alpha}(x, y)\right|^{1 / \alpha} \leq \sqrt{e_{\alpha}(x)^{1 / \alpha} e_{\alpha}(y)^{1 / \alpha}}
$$

Owing to (2) and the hypothesis of local boundedness of $1 / F$, it follows that $\left|K_{\alpha}(x, y)\right|^{1 / \alpha}$ is locally bounded on $\Omega \times \Omega$, and uniformly so when $\alpha$ ranges through $[1,+\infty)$.

Now let $\alpha_{1}<\alpha_{2}<\ldots$ be a sequence of numbers from $A$ which tend to infinity. Since $K_{\alpha_{j}}(x, y) \neq 0$ on $U \times U$, it follows from the simple connectivity of $U$ that there exists a single-valued holomorphic branch of $\log K_{\alpha_{j}}(x, \bar{y}), x, \bar{y} \in U$; we can choose this branch to be real on the diagonal $x=\bar{y}$. Define $f_{j}=K_{\alpha_{j}}^{1 / \alpha_{j}}=\exp \left(\frac{1}{\alpha_{j}} \log K_{\alpha_{j}}\right)$. By the preceding observation, $f_{j}(x, y)$ is a locally uniformly bounded family of sesqui-holomorphic (i.e. holomorphic in $x$ and anti-holomorphic in $y$ ) functions on $U \times U$. A standard normal family argument shows that there exists a subsequence $f_{j_{k}}$ which converges to a sesqui-holomorphic function $f$ uniformly on compact subsets of $U \times U$. For $x=y$, we must have $f(x, x)=\rho(x)=1 / F(x)$ by hypothesis. Since each $f_{j}$ is zero-free, it follows from the Hurwitz theorem ([24], Theorem 3.4.5) - which is easily adapted to the case of several complex variables - that $f$ is either zero-free or identically zero; the latter possibility is, however, ruled out since $1 / F \neq \mathbf{0}$. Finally, setting $\alpha=\alpha_{j_{k}}$ and taking the limit as $k \rightarrow \infty$, we see from (10) that

$$
|f(x, y)|^{2} \leq f(x, x) f(y, y) .
$$


Thus, the function $F(x, y)=1 / f(x, y)$ has all the properties required by the theorem.

\section{SOME EXAMPLES}

Example 1. Let $F, G$ be such that $A_{\alpha}^{2}=\{0\}$ for all $\alpha$; e.g. $\Omega=\mathbb{C}, G=\mathbf{1}, F=\mathbf{1}$. Then $e_{\alpha}(x)=\mathbf{0}$, hence $\lim _{\alpha \rightarrow+\infty} e_{\alpha}(x)^{1 / \alpha} \equiv \rho=\mathbf{0}$. This trivial example shows that some additional hypothesis is required to ensure that $\rho(x)=1 / F(x)$. Moreover, $F^{* *}=F^{* * *}=F^{\#}=F=\mathbf{1}$ in this case, so we also see that the hypothesis that $\mathbf{1} \in A_{\alpha}^{2}$ for some $\alpha>0$ in Theorem B cannot be omitted.

In the remaining examples (except the very last one), we consider the case when $\Omega$ is the unit disc $\mathbb{D}$ or the complex plane $\mathbb{C}$, and $F(z)$ and $G(z)$ are radial functions, i.e. functions depending only on the modulus $|z|$ :

$$
\begin{aligned}
& F(z)=\Phi\left(|z|^{2}\right), \\
& G(z)=\gamma\left(|z|^{2}\right) .
\end{aligned}
$$

It is then easily verified by passing to polar coordinates (cf. [23], Theorem 0.8, or [11], Proposition 3.11) that

$$
\|f\|_{\alpha}^{2}=\sum_{n=0}^{\infty}\left|f_{n}\right|^{2} \cdot\left(\pi \int_{0}^{B} t^{n} \Phi(t)^{\alpha} \gamma(t) d t\right),
$$

where $f_{n}$ are the Taylor coefficients of $f$ and $B=1$ or $+\infty$ for $\Omega=\mathbb{D}$ and $\mathbb{C}$, respectively; moreover, the reproducing kernels are given by

$$
K_{\alpha}(x, y)=\sum_{n=0}^{\infty}(x \bar{y})^{n} /\left(\pi \int_{0}^{B} t^{n} \Phi^{\alpha} \gamma d t\right)
$$

with the convention that $1 /+\infty=0$.

Note that the last series converges for

$$
|x \bar{y}|<\sup \left\{t: t \in \operatorname{support}\left(\Phi^{\alpha} \gamma d t\right)\right\} .
$$

Indeed, the radius of convergence for a series $\sum_{0}^{\infty} z_{n} / c_{n}$ is equal to $\liminf c_{n}^{1 / n}$, and by the familiar result from abstract measure theory (already alluded to in Section 2), valid for any measure space,

$$
\lim _{n \rightarrow \infty}\|h\|_{n}= \begin{cases}\|h\|_{\infty} & \text { if } \exists \text { finite } p_{0}: h \in L^{p} \forall p \in\left(p_{0}, \infty\right), \\ +\infty & \text { otherwise, }\end{cases}
$$

where $\|h\|_{p}$ is the $L_{p}$ norm of a function $h$.

Example 2. $\Omega=\mathbb{D}, G=\mathbf{1} ; F(z)=\Phi\left(|z|^{2}\right)$, where $\Phi$ is continuous on $[0,1]$, $0<\Phi \leq \Phi(1)=1$. By the result of the preceding section,

$$
\limsup _{\alpha \rightarrow+\infty} e_{\alpha}(x)^{1 / \alpha} \equiv \bar{\rho}(x) \leq 1 / \Phi\left(|x|^{2}\right) .
$$

It is immediate from (11) that $e_{\alpha}(x)=K_{\alpha}(x, x)$ is a non-decreasing function of $|x|^{2}$. Hence, the same is true for $e_{\alpha}(x)^{1 / \alpha}$ and for the limit $\bar{\rho}(x)$. Thus

$$
\bar{\rho}(x) \leq \lim _{|x| \rightarrow 1} \bar{\rho}(x) \leq \lim _{|x| \rightarrow 1} \Phi\left(|x|^{2}\right)^{-1}=1 .
$$


On the other hand, $\Phi \leq 1$ implies that

$$
\int_{0}^{1} t^{n} \Phi^{\alpha} d t \leq \frac{1}{n+1}
$$

and

$$
e_{\alpha}(x) \geq \pi^{-1}\left(1-|x|^{2}\right)^{-2}
$$

so $\liminf \inf _{\alpha \rightarrow+\infty} e_{\alpha}(x)^{1 / \alpha} \geq 1$. Thus the limit $\rho(x)$ exists and

$$
\rho(x)=\lim _{\alpha \rightarrow+\infty} e_{\alpha}(x)^{1 / \alpha}=1,
$$

regardless of the choice of $\Phi$.

This shows that the map $F \mapsto \rho$ (defined for those $F$ for which the limit $\rho$ exists) is not injective.

Note that $F^{*}=F^{* *}=\mathbf{1}$ by the maximum principle. So in this case, $\rho(x)$ exists and is equal to $1 / F^{*}<1 / F$ :

$$
1 / F^{*}=1 / F^{* *}=1 / F^{* * *}=\rho=1 / F^{\#}<1 / F .
$$

In general, for any $\Phi$ continuous on $[0,1]$ it follows from $(11)$ that $\rho(x)$, if it exists, must be a non-decreasing function of $|x|$; thus a necessary condition for $\rho=1 / F$ is that $\Phi$ be non-increasing. As we shall shortly see, even this condition is far from sufficient; still, observe that it implies that (granted $1 \in A_{\alpha}^{2}$ for some $\alpha$ )

$$
\lim _{\alpha \rightarrow+\infty} K_{\alpha}(0,0)^{1 / \alpha}=1 / \lim _{\alpha \rightarrow+\infty}\|\Phi\|_{L^{\alpha}(\gamma d t)}=1 /\|\Phi\|_{\infty}=1 / \Phi(0),
$$

i.e. one has at least $\rho(0)=1 / F(0)$. If $\Phi$ is $C^{\infty}$ on $[0,1)$ and has a strict maximum at the origin, much more precise information about the asymptotic behaviour of $K_{\alpha}(0,0)^{1 / \alpha}$ can be extracted from (11) by means of the familiar Laplace method (see e.g. [14], § II.1).

Example 3. $\Omega=\mathbb{C}, G=\mathbf{1}, F(z)=\Phi\left(|z|^{2}\right)$, where

$$
\Phi(t)= \begin{cases}A, & 0 \leq t \leq 1+1 / A \\ \frac{1}{t-1}, & t \geq 1+1 / A\end{cases}
$$

$A$ being a positive constant. The integrals in (11) are equal to

$$
\int_{0}^{+\infty} t^{n} \Phi^{\alpha} d t \equiv c_{n}=\int_{0}^{1+1 / A}+\int_{1+1 / A}^{+\infty} \equiv J_{n, \alpha}+I_{n, \alpha} .
$$

Computation gives

$$
J_{n, \alpha}=A^{\alpha} \cdot\left(1+\frac{1}{A}\right)^{n+1} \cdot \frac{1}{n+1}
$$

and

$$
I_{0, \alpha}=\frac{A^{\alpha-1}}{\alpha-1} \quad(\alpha>1), \quad I_{n, \alpha}=I_{n-1, \alpha}+I_{n-1, \alpha-1},
$$

from which it follows that

$$
I_{n, \alpha}=\sum_{j=0}^{n}\left(\begin{array}{l}
n \\
j
\end{array}\right) \frac{A^{\alpha-1-j}}{\alpha-1-j}, \quad \text { if } 0 \leq n \leq \alpha-1,
$$


and $I_{n, \alpha}=+\infty$ otherwise. Now on the one hand

$$
c_{n} \geq I_{n, \alpha} \geq \frac{1}{\alpha-1} \sum_{j=0}^{n}\left(\begin{array}{l}
n \\
j
\end{array}\right) A^{\alpha-1-j}=\frac{A^{\alpha-1}}{\alpha-1}\left(1+\frac{1}{A}\right)^{n}
$$

and, for any $t \geq 0$,

$$
\begin{aligned}
\sum_{0 \leq n<\alpha-1} t^{n} / c_{n} & \leq \frac{\alpha-1}{A^{\alpha-1}} \sum_{0 \leq n<\alpha-1}\left(\frac{t}{1+\frac{1}{A}}\right)^{n} \\
& \leq \frac{(\alpha-1) \alpha}{A^{\alpha-1}} \cdot\left[\max \left(1, \frac{t}{1+\frac{1}{A}}\right)\right]^{\alpha-1} .
\end{aligned}
$$

It follows that

$$
\limsup _{\alpha \rightarrow+\infty} e_{\alpha}(x)^{1 / \alpha} \leq \frac{1}{A} \max \left(1, \frac{|x|^{2}}{1+\frac{1}{A}}\right) .
$$

On the other hand, for $0 \leq n<\alpha-2$ we have

$$
\begin{aligned}
c_{n} & \leq \sum_{j=0}^{n}\left(\begin{array}{c}
n \\
j
\end{array}\right) \frac{A^{\alpha-1-j}}{\alpha-1-n}+J_{n, \alpha} \\
& =A^{\alpha-1}\left(1+\frac{1}{A}\right)^{n} \cdot\left[\frac{1}{\alpha-1-n}+\frac{A+1}{n+1}\right] \\
& \leq A^{\alpha-1}\left(1+\frac{1}{A}\right)^{n} \cdot(A+2)
\end{aligned}
$$

and, for any $t \geq 0$,

$$
\begin{aligned}
\sum_{0 \leq n<\alpha-1} t^{n} / c_{n} & \geq \sum_{0 \leq n<\alpha-2} t^{n} / c_{n} \\
& \geq \frac{A^{1-\alpha}}{A+2} \sum_{0 \leq n<\alpha-2}\left(\frac{t}{1+\frac{1}{A}}\right)^{n} \\
& \geq \frac{A^{1-\alpha}}{A+2} \cdot\left[\max \left(1, \frac{t}{1+\frac{1}{A}}\right)\right]^{\alpha-3}
\end{aligned}
$$

for $\alpha \geq 3$. Consequently,

$$
\liminf _{\alpha \rightarrow+\infty} e_{\alpha}(x)^{1 / \alpha} \geq \frac{1}{A} \max \left(1, \frac{|x|^{2}}{1+\frac{1}{A}}\right) .
$$

Thus we conclude that

$$
\rho(x) \equiv \lim _{\alpha \rightarrow+\infty} e_{\alpha}(x)^{1 / \alpha}=\frac{1}{A} \max \left(1, \frac{|x|^{2}}{1+\frac{1}{A}}\right)= \begin{cases}1 / A & \text { if } 0 \leq|x|^{2} \leq 1+1 / A, \\ \frac{|x|^{2}}{A+1} & \text { if }|x|^{2} \geq 1+1 / A,\end{cases}
$$

and we see that $\rho(x)=1 / F(x)$ for $0 \leq|x|^{2} \leq 1+1 / A$, but $\rho(x)<1 / F(x)$ for $|x|^{2}>1+1 / A$.

It can be shown that $F^{*}=F^{* *} \equiv A$ in this case. (Use the Borel-Carathéodory theorem (see $\S 5.5$ in [24]), or just plain Cauchy estimates.) Also, taking $\varkappa=2$ and $f(z)=z / \sqrt{A+1}$ shows that $F^{* * *}=1 / \rho$.

Note also that the function $-\log F$ is not convex (so this example does not contradict Theorem A). In fact, it is not even subharmonic, and we finish by showing 
that its greatest subharmonic minorant $-\log F^{\#}$ is also equal to $\log \rho$, so that we have

$$
\mathbf{1}=F^{*}=F^{* *} \supsetneqq F^{* * *}=\frac{1}{\rho}=F^{\#} \supsetneqq F .
$$

We already know that $\log \rho$ is subharmonic, so assume that $\psi$ is a subharmonic function satisfying $\log \rho \leq \psi \leq \phi:=-\log F$. Then $\psi \equiv-\log A$ on the $\operatorname{disc}$ $|z|^{2} \leq R:=1+1 / A$, so it suffices to deal with the region $|z|^{2}>R$. Let

$$
\chi(x):=\log |x|^{2}+\psi(\sqrt{R} / x), \quad x \in \mathbb{D} \backslash\{0\} .
$$

Since the inversion $x \mapsto \sqrt{R} / x$ preserves (sub)harmonicity and $\log |x|^{2}$ is harmonic on the punctured disc, we see that $\chi$ is a subharmonic function on $\mathbb{D} \backslash\{0\}$ which satisfies

$\log |x|^{2}+\log \rho(\sqrt{R} / x)=\log (R-1) \leq \chi(x) \leq \log |x|^{2}+\phi(\sqrt{R} / x)=\log \left(R-|x|^{2}\right)$.

A standard maximum principle argument implies that

$$
0 \leq \chi(x)-\log (R-1) \leq \frac{\log |x|^{2}}{\log \epsilon} \log \frac{R-\epsilon}{R-1}
$$

on the annulus $\epsilon \leq|x|^{2} \leq 1$, for any $1>\epsilon>0$. Thus $\chi \equiv \log (R-1)$, or $\psi\left(\frac{\sqrt{R}}{x}\right)=\log \frac{R-1}{|x|^{2}}=\log \rho\left(\frac{\sqrt{R}}{x}\right)$, so $\psi=\log \rho$ and the assertion follows.

Example 4. $\Omega=\mathbb{C}, G=\mathbf{1}, F(x)=\Phi\left(|x|^{2}\right)$, where

$$
\Phi(t)= \begin{cases}1, & 0 \leq t \leq 1 \\ 1 / t, & t \geq 1\end{cases}
$$

Proceeding as in the preceding example, we get

$$
\int_{0}^{\infty} t^{n} \Phi^{\alpha} d t= \begin{cases}\frac{1}{n+1}-\frac{1}{n-\alpha+1} & \text { if } 0 \leq n<\alpha-1 \\ +\infty & \text { otherwise }\end{cases}
$$

Thus

$$
e_{\alpha}(x)=\frac{1}{\pi} \sum_{0 \leq n<\alpha-1} \frac{(n+1)(\alpha-n-1)}{\alpha}|x|^{2 n} .
$$

As before, it is easy to obtain the estimates

$$
\begin{aligned}
& \pi e_{\alpha}(x) \leq \frac{1}{\alpha} \cdot \alpha^{2} \sum_{0 \leq n<\alpha-1}|x|^{2 n} \leq \alpha^{2}\left[\max \left(1,|x|^{2}\right)\right]^{\alpha}, \\
& \pi e_{\alpha}(x) \geq \frac{1}{\alpha} \cdot 1(\alpha-1) \sum_{0 \leq n<\alpha-2}|x|^{2 n} \geq \frac{\alpha-1}{\alpha}\left[\max \left(1,|x|^{2}\right)\right]^{\alpha-3} .
\end{aligned}
$$

It follows that the limit $\rho(x)$ exists and equals

$$
\rho(x) \equiv \lim _{\alpha \rightarrow+\infty} e_{\alpha}(x)^{1 / \alpha}=\max \left(1,|x|^{2}\right)=1 / F .
$$

On the other hand, if $e^{g} \equiv f$ satisfies $|f|^{2} \leq 1 / F$, then $f$ is an entire function satisfying

$$
|f(z)| \leq \max (1,|z|) .
$$

In view of the Cauchy estimates, this implies that the Taylor coefficients $f_{n}$ of $f$ vanish for $n>1$. Thus $f(z)=f_{1} z+f_{0}$, and as $f=e^{g}$ is necessarily zero-free, we 
must have $f(z)=f_{0} \equiv$ const. It follows that $F^{* *}=F^{*}=\mathbf{1} \neq F$. Also, putting $\varkappa=2$ and $f(z)=z$ in the definition shows that $F^{* * *}=F$, so, summarizing,

$$
\mathbf{1}=F^{*}=F^{* *} \supsetneqq F^{* * *}=1 / \rho=F^{\#}=F .
$$

This time, we see that $\rho=1 / F$, even though $F^{*} \neq F$ and $-\log F$ is not convex. Observe, however, that the function $-\log F$ is, at least, subharmonic in this case.

The next two examples are concerned with the convergence of $K_{\alpha}(x, y)$ on all of $\Omega \times \Omega$ (i.e. not only on the diagonal $x=y$ ).

Example 5. $\Omega=\mathbb{D}, G(x)=1 /|x|=\gamma\left(|x|^{2}\right), F(x)=1-|x|=\Phi\left(|x|^{2}\right)$, where $\gamma(t)=1 / \sqrt{t}, \Phi(t)=1-\sqrt{t}$. We claim that $-\log F$ is a convex function. Indeed, in general, it is well-known that a real-valued, twice continuously differentiable function $f(z)$ defined on a region in the plane is convex if and only if the $2 \times 2$ hermitian matrix

$$
\left(\begin{array}{cc}
\frac{\partial^{2} f}{\partial z \partial \bar{z}} & \frac{\partial^{2} f}{\partial z^{2}} \\
\frac{\partial^{2} f}{\partial \bar{z}^{2}} & \frac{\partial^{2} f}{\partial z \partial \bar{z}}
\end{array}\right)
$$

is positive semidefinite. The latter condition can also be written as

$$
\frac{\partial^{2} f}{\partial z \partial \bar{z}} \geq\left|\frac{\partial^{2} f}{\partial z^{2}}\right|
$$

If, in particular, $f(z)=\phi\left(|z|^{2}\right)$ is a radial function, this reads

$$
\left(t \phi^{\prime}\right)^{\prime} \geq\left|t \phi^{\prime \prime}\right|
$$

or

$$
\phi^{\prime} \geq 0 \quad \text { and } \quad \phi^{\prime}+2 t \phi^{\prime \prime} \geq 0 .
$$

In our case $\phi(t)=-\log (1-\sqrt{t})$, so

$$
\phi^{\prime}=\frac{1}{2 \sqrt{t}(1-\sqrt{t})}>0, \quad \phi^{\prime}+2 t \phi^{\prime \prime}=\frac{1}{2(1-\sqrt{t})^{2}}>0,
$$

and the claim follows.

By Theorem A, the limit $\rho(x)$ exists and equals $1 / F(x)$.

On the other hand, the function $F(x)$ clearly cannot be extended to a function $F(x, y)$ such that $F(x)=F(x, x)$ and $F(x, \bar{y})$ is holomorphic on $\mathbb{D} \times \mathbb{D}$. The only possible candidate is $F(x, y)=1-\sqrt{x \bar{y}}$, which is not well defined on $\mathbb{D} \times \mathbb{D}$; however, a single-valued branch exists on $U \times U$ for any simply-connected subregion $U$ of $\mathbb{D}$ not containing the origin. By Theorem $\mathrm{C}$, we conclude that for all sufficiently large $\alpha, K_{\alpha}(x, y)$ must have a zero at some point and, moreover, these zeroes accumulate at the origin.

In a simple case like this we can verify the last claim directly. Using again the formula (11), a computation shows that (cf. [11], Example 3.31)

$$
\begin{aligned}
K_{\alpha}(x, y) & =\frac{1}{2 \pi} \sum_{n=0}^{\infty} \frac{\Gamma(2 n+\alpha+2)}{\Gamma(2 n+1) \Gamma(\alpha+1)}(x \bar{y})^{n} \\
& =\frac{\alpha+1}{4 \pi}\left[(1-\sqrt{x \bar{y}})^{-\alpha-2}+(1+\sqrt{x \bar{y}})^{-\alpha-2}\right] .
\end{aligned}
$$


(Note that this is a single-valued holomorphic function of $x \bar{y}$, even though $\sqrt{x \bar{y}}$ itself is not!) It follows that for any integer $k$ and

$$
x \bar{y}=-\tan ^{2}\left(k+\frac{1}{2}\right) \frac{\pi}{\alpha}=\tanh ^{2} \frac{(2 k+1) \pi i}{2 \alpha}
$$

we have

$$
\frac{1+\sqrt{x \bar{y}}}{1-\sqrt{x \bar{y}}}=e^{ \pm(2 k+1) \pi i / \alpha}
$$

and therefore $K_{\alpha-2}(x, y)=0$.

Example 6. $\Omega=\mathbb{D}, G=\mathbf{1}$, and $F(x)=\Phi\left(|x|^{2}\right)$, where $\Phi$ is the polynomial

$$
\Phi(t)=(t-1)\left(t+\frac{3}{4}\right)\left(t-\frac{11}{4}\right) .
$$

The function $\phi=-\log \Phi$ satisfies

$$
-\phi^{\prime}=\frac{\Phi^{\prime}}{\Phi}=\frac{1}{t-1}+\frac{1}{t+\frac{3}{4}}+\frac{1}{t-\frac{11}{4}}<0 \quad \text { on }[0,1],
$$

since $\Phi>0$ and $\Phi^{\prime}<0$ on this interval (cf. [11], Example 3.25); and

$$
\phi^{\prime \prime}=\frac{1}{(t-1)^{2}}+\frac{1}{\left(t+\frac{3}{4}\right)^{2}}+\frac{1}{\left(t-\frac{11}{4}\right)^{2}}>0,
$$

so $\phi^{\prime}+2 t \phi^{\prime \prime} \geq 0$ on $[0,1]$. As in the preceding example, it follows that $-\log F$ is a convex function, and as $\Phi$ is bounded, Theorem A applies. By Theorem C, if there existed a sequence $\alpha_{j} \rightarrow \infty$ such that each $K_{\alpha_{j}}(x, y)$ were zero-free on $\mathbb{D} \times \mathbb{D}$, then

$$
\lim _{j \rightarrow \infty} K_{\alpha_{j}}(x, y)^{1 / \alpha_{j}}=1 / F(x, y),
$$

where $F(x, y)$ would be a sesqui-holomorphic extension of $F(x)$ to $\mathbb{D} \times \mathbb{D}$. The only possible such extension is given by (cf. [7], Theorem II.7)

$$
F(x, y)=(x \bar{y}-1)\left(x \bar{y}+\frac{3}{4}\right)\left(x \bar{y}-\frac{11}{4}\right) .
$$

However, taking $x=-y=\sqrt{3} / 2$ gives $F(x, y)=0$, so $F(x, y)$ is not zero-free, and

$$
0=|F(x, y)|^{2}<F(x, x) F(y, y)=\frac{9}{16}
$$

so the "reverse Schwarz" inequality is likewise violated. It follows that for all sufficiently large $\alpha, K_{\alpha}(x, y)$ must have a zero.

Example 7. In this final example we exhibit a situation in which $\bar{\rho} \supsetneqq 1 / F^{* * *}$. (In other words, the assertion of Theorem $\mathrm{B}^{\prime}$ is not the sharpest one possible.) To that end, consider the function (8):

$$
\phi(x)=\sum_{j=2}^{\infty} 2^{-j} \log \left|b_{j}(x)\right|^{2}, \quad b_{j}(x):=\frac{x-1 / j}{1-x / j} .
$$

Clearly $\phi$ is subharmonic (hence, upper semicontinuous) and

$$
\phi(1 / j)=-\infty, \quad j=2,3, \ldots,
$$

while, on the other hand,

$$
\phi(0)=-2 \sum_{j=2}^{\infty} \frac{\log j}{2^{j}}>-\infty .
$$


Pick a number $\epsilon, 0<\epsilon<e^{\phi(0)}$, and let

$$
\phi_{\epsilon}=\max (\phi, \log \epsilon) .
$$

Now take $\Omega=\mathbb{D}, G=\pi^{-1} \mathbf{1}$ and $F=e^{-\phi_{\epsilon}}$. Owing to (*), any continuous function $\Psi$ lying below $1 / F$ must satisfy $\Psi(1 / j) \leq \epsilon, j=2,3, \ldots$, and, hence, also $\Psi(0) \leq \epsilon$. It follows that

$$
1 / F^{*}(0)=1 / F^{* *}(0)=1 / F^{* * *}(0)=\epsilon<e^{\phi(0)}=1 / F(0)=1 / F^{\#}(0) .
$$

Let us now obtain a bound for $\bar{\rho}(0)$. By $(4)$,

$$
e_{\alpha}(0)^{1 / \alpha} \geq \frac{|f(0)|^{2 / \alpha}}{\|f\|_{\alpha}^{2 / \alpha}}
$$

for any analytic function $f$ which does not vanish identically. Let us take $\alpha=2^{n}$ and

$$
f(x)=\prod_{j=2}^{n} b_{j}(x)^{2^{n-j}} .
$$

We have $|f(0)|^{2 / 2^{n}}=\exp \left(-2 \sum_{j=2}^{n} 2^{-j} \log j\right)$, which tends to $e^{\phi(0)}$ if $n$ goes to infinity. On the other hand,

$$
\begin{aligned}
\|f\|_{2^{n}}^{2 / 2^{n}} & =\left(\int_{\mathbb{D}}|f|^{2} \exp \left(-2^{n} \phi_{\epsilon}\right) d \mu\right)^{1 / 2^{n}} \\
& =\left(\int_{\mathbb{D}} \frac{\prod_{j=2}^{n}\left|b_{j}(x)\right|^{2^{n+1-j}}}{\max \left(\prod_{j=2}^{\infty}\left|b_{j}(x)\right|^{2^{n+1-j}}, \epsilon^{2^{n}}\right)} d \mu(x)\right)^{1 / 2^{n}} \\
& =\left(\int_{\mathbb{D}} \min \left(\frac{\prod_{j=2}^{n}\left|b_{j}\right|^{2^{n+1-j}}}{\epsilon^{2^{n}}}, \frac{1}{\prod_{j=n+1}^{\infty}\left|b_{j}\right|^{2^{n+1-j}}}\right) d \mu\right)^{1 / 2^{n}} \equiv\left\|f_{n}\right\|_{L^{2^{n}}(d \mu)},
\end{aligned}
$$

where

$$
f_{n}:=\min \left(\frac{\prod_{j=2}^{n}\left|b_{j}\right|^{2^{1-j}}}{\epsilon}, \frac{1}{\prod_{j=n+1}^{\infty}\left|b_{j}\right|^{2^{1-j}}}\right) .
$$

Thus we have arrived at

$$
\bar{\rho}(0) \geq \frac{e^{\phi(0)}}{\lim _{n \rightarrow \infty}\left\|f_{n}\right\|_{L^{2^{n}}(d \mu)}} .
$$

We claim that the limit in the denominator equals one. To see this, observe first of all that $f_{n+1}=\left|b_{n+1}\right|^{2^{-n}} f_{n}$, so by the standard property of the Blaschke products $f_{n+1} \leq f_{n}$. Hence, $f_{n} \geq f_{n+1} \geq f_{n+2} \geq \cdots \geq f_{\infty}$, where

$$
f_{\infty}:=\lim _{n \rightarrow \infty} f_{n}=\min \left(e^{\phi} / \epsilon, 1\right)
$$

Owing to the finiteness of $d \mu$, it therefore follows that

$$
\left\|f_{n}\right\|_{L^{2^{n}}(d \mu)} \geq\left\|f_{\infty}\right\|_{L^{2^{n}}(d \mu)} \rightarrow\left\|f_{\infty}\right\|_{\infty} \geq f_{\infty}(0)=\min \left(\frac{e^{\phi(0)}}{\epsilon}, 1\right)=1 .
$$

On the other hand, since the convergence of the sum (8) is locally uniform as long as we stay away from the points $j$ and $1 / j(j=2,3, \ldots)$, the functions $\phi, f_{n}$ and $f_{\infty}$ extend continuously to the boundary of the unit disc, and $f_{n}=f_{\infty}=1$ there. By Dini's theorem, $f_{n} \searrow f_{\infty}$ therefore implies $\left\|f_{n}\right\|_{\infty} \rightarrow\left\|f_{\infty}\right\|_{\infty}$, and, further, the 
fact that $d \mu$ is a probability measure implies that $\|\cdot\|_{L^{p}(d \mu)}$ is a nondecreasing function of $p$, by Hölder's inequality; consequently,

$$
\left\|f_{n}\right\|_{L^{2^{n}}(d \mu)} \leq\left\|f_{n}\right\|_{\infty} \rightarrow\left\|f_{\infty}\right\|_{\infty}=\left\|\min \left(e^{\phi} / \epsilon, 1\right)\right\|_{\infty} \leq 1 .
$$

Thus, indeed, $\lim _{n \rightarrow \infty}\left\|f_{n}\right\|_{L^{2^{n}(d \mu)}}=1$, and

$$
\bar{\rho}(0) \geq e^{\phi(0)} .
$$

Hence $\bar{\rho}(0)=e^{\phi(0)}$. Summing everything up, we see that in this case

$$
1 / F^{*}(0)=1 / F^{* *}(0)=1 / F^{* * *}(0)<\bar{\rho}(0)=1 / F^{\#}(0)=1 / F(0),
$$

as we have asserted.

\section{Postscript: A few open problems}

(I) The author does not know of any situation in which the limit $\rho(x)$ would fail to exist. Is it true that this limit always exists?

(II) If the answer to (I) is affirmative, is there a neat formula for the limit? For instance, can it be true that

$$
\rho=1 / F^{\#}
$$

whenever $\mathbf{1} \in A_{\alpha}^{2}$ for some $\alpha$ ? Note that this gives the correct answer in all the examples above.

(III) Characterize the functions $F$ for which (a) $F=F^{* *}$, or (b) $F=F^{* * *}$. In other words, give an "easy" criterion for a function $\phi=-\log F$ to belong to $\mathcal{H}_{0}^{\text {sup }}(\Omega)$ or $\mathcal{G}^{\text {sup }}(\Omega)$, in the notation $(6)$.

(IV) Adding yet another definition to (6), let

$$
\mathcal{G}^{\infty}(\Omega):=\left\{\sum_{j=1}^{\infty} \varkappa_{j} \log \left|f_{j}\right|: f_{j} \text { holomorphic on } \Omega, \varkappa_{j}>0\right\},
$$

and

$$
\phi^{\infty}:=\sup \left\{\psi: \psi \in \mathcal{G}^{\infty}, \psi \leq \phi\right\} .
$$

Is it true that $\phi=\phi^{\infty}$ for any PSH function $\phi$ ? (Observe that if we used only finite sums in the definition of $\mathcal{G}^{\infty}$, then, by an easy approximation argument, $\phi^{\infty}$ would be just the same thing as $\phi^{\mathcal{G}}$.)

It would also be of interest to clarify the relation between $\mathcal{G}^{\text {sup }}$ and $P S H$.

(V) In the applications in quantization, $\Omega$ is a Kähler manifold whose Kähler metric $d s^{2}=g_{i \bar{j}} d z^{i} d \bar{z}^{j}$ is given by (in local coordinates)

$$
g_{i \bar{j}}=\frac{\partial^{2} \Psi}{\partial z^{i} \partial \bar{z}^{j}},
$$

where $\Psi$ is a real-valued function on $\Omega$ (the Kähler potential); one then takes $F=e^{-\Psi}$ and $G=\operatorname{det}\left(g_{i \bar{j}}\right)$ (the volume form). Thus, in view of the positivedefiniteness of the metric tensor $g_{i \bar{j}}$, the function $-\log F=\Psi$ is automatically strictly plurisubharmonic. What are the Kähler manifolds for which $F=F^{*}$ ? What are the ones for which $\Psi$ belongs to $\mathcal{H}_{0}^{\text {sup }}(\Omega)$ or $\mathcal{G}^{\text {sup }}(\Omega)$ ?

(VI) In the applications to quantization, one further needs something stronger than the equality $\rho(x)=1 / F(x)$ or even $\lim K_{\alpha}(x, y)^{1 / \alpha}=1 / F(x, y)$. What is 
needed is that

$$
\lim _{j \rightarrow \infty} \frac{K_{\alpha_{j}}(x, y) F(x, y)^{\alpha_{j}}}{\alpha_{j}^{\operatorname{dim} \Omega}}=\mathbf{1}
$$

for some sequence $\alpha_{j}$ tending to infinity. (The numbers $1 / \alpha_{j}$ then correspond to the admissible values of the Planck constant.) This presupposes that $F(x)=F(x, x)$ for some sesqui-holomorphic function $F(x, y)$ on $\Omega \times \Omega$, and that $\rho(x)$ exists and equals $1 / F(x)$. It would be desirable to strengthen the results of the present paper so as to obtain (12) instead of (1).

(VII) Observe that the case $G=\mathbf{1}$ (or, upon replacing $\alpha$ by $\alpha-\beta$, which has no influence on the limit $\rho(x), G=F^{\beta}$ for some real $\beta$ ) corresponds to the case when the metric $g_{i \bar{j}}$ has "constant curvature" - more precisely: when it is a Kähler-Einstein metric. Can some of the problems above be solved at least in this important case? It is known that a complete Kähler-Einstein metric exists e.g. on any bounded pseudoconvex domain in $\mathbb{C}^{n}$, and is unique (up to rescaling) if the domain is strongly pseudoconvex (see, for instance, the survey article by Wu [29]).

We remark that the completeness of the metric corresponds to the function $F$ having a zero on $\partial \Omega$ of precisely the first order. Thus dealing with complete metrics automatically rules out such pathological situations as in Example 2.

\section{REFERENCES}

1. V. Bargmann, On a Hilbert space of analytic functions and an associated integral transform, Comm. Pure Appl. Math. 14 (1961), 187-214. MR 28:486

2. H. Bauer, Approximation and abstract boundaries, Amer. Math. Monthly 85 (1978), 632-647. MR 80f: 41014

3. F.A. Berezin, Quantization, Math. USSR Izvestiya 8 (1974), 1109-1163. MR 52:16404

4. C.A. Berger, L.A. Coburn, Toeplitz operators and quantum mechanics, J. Funct. Anal. 68 (1986), 273-299. MR 88b:46098

5. C.A. Berger, L.A. Coburn, Toeplitz operators on the Segal-Bargmann space, Trans. Amer. Math. Soc. 301 (1987), 813-829. MR 88c:47044

6. S. Bergman, The kernel function and conformal mapping, $2^{\text {nd }}$ edition, American Mathematical Society, Providence, 1970. MR 58:22502

7. S. Bochner, W.T. Martin, Several complex variables, Princeton University Press, Princeton, 1948. MR 10:366a

8. M. Cahen, S. Gutt, J. Rawnsley, Quantization of Kähler manifolds. I: Geometric interpretation of Berezin's quantization, J. Geom. Physics 7 (1990), 45-62; II, Trans. Amer. Math. Soc. 337 (1993), 73-98; III, Letters in Math. Phys. 30 (1994), 291-305. MR 92e:58082; MR 93i:58063; MR 95c:58082

9. M. Engliš, Asymptotics of reproducing kernels on a plane domain, Proc. Amer. Math. Soc. 123 (1995), 3157-3160. MR 95m:30013

10. M. Engliš, Asymptotics of the Berezin transform and quantization on planar domains, Duke Math. J. 79 (1995), 57-76. MR 96m:47045

11. M. Engliš, Berezin quantization and reproducing kernels on complex domains, Trans. Amer. Math. Soc. 348 (1996), 411-479. MR 96j:32008

12. J. Faraut, A. Korányi, Function spaces and reproducing kernels on bounded symmetric domains, J. Funct. Anal. 88 (1990), 64-89. MR 90m:32049

13. J. Faraut, A. Korányi, Analysis on symmetric cones, Clarendon Press, Oxford, 1994.

14. M.V. Fedoryuk, Asymptotics, integrals, series, Nauka, Moscow, 1987 (in Russian). MR 89j: 41045

15. S. Helgason, Differential geometry and symmetric spaces, Academic Press, New York, 1962. MR 26:2986

16. L.-K. Hua, Harmonic analysis of functions of several complex variables in the classical domains, American Mathematical Society, Providence, 1963. MR 30:2162 
17. S. Janson, J. Peetre, R. Rochberg, Hankel forms and the Fock space, Revista Math. Iberoamer. 3 (1987), 61-138. MR 91a:47029

18. M. Klimek, Pluripotential theory, Clarendon Press, Oxford, 1991. MR 93h:32021

19. I. Kra, Automorphic functions and Kleinian groups, Benjamin, Reading, 1972. MR 50:10242

20. O. Loos, Bounded symmetric domains and Jordan pairs, University of California, Irvine, 1977.

21. I. Netuka, J. Veselý, On harmonic functions. Solution to problem 6393 [1982, 502] proposed by G.A. Edgar, Amer. Math. Monthly 91 (1984), 61-62.

22. W. Rudin, Function theory in the unit ball of $\mathbb{C}^{n}$, Springer Verlag, Berlin-Heidelberg-New York, 1980. MR 82i:32002

23. M. Skwarczynski, Biholomorphic invariants related to the Bergman kernel function, Dissert. Math. 173 (1980). MR 82e:32038

24. E.C. Titchmarsh, The theory of functions, Oxford University Press, Oxford, 1939.

25. A. Unterberger, H. Upmeier, The Berezin transform and invariant differential operators, Comm. Math. Phys. 164 (1994), 563-598. MR 96h:58170

26. H. Upmeier, Weyl quantization of complex domains, in: Operator algebras and topology, Craiova 1989 (Pitman Research Notes in Math. 270), Longman Scientific and Technical, Harlow, 1992, pp. 160-178. MR 93e:00027

27. Z. Vondracek, On some extremal elements in the cone $\mathcal{H}^{\mathrm{inf}}$, Glas. Mat. Ser. III 27(47) (1992), 241-249. MR 94i:31006

28. Z. Vondracek, The Martin kernel and infima of positive harmonic functions, Trans. Amer. Math. Soc. 335 (1993), 547-557. MR 93d:31009

29. H. Wu, Old and new invariant metrics on complex manifolds, in: J.E. Fornaess (editor): Several complex variables, Proceedings of the Mittag-Leffler Institute, Stockholm 1987/1988 (Math. Notes, vol. 38), Princeton University Press, Princeton, 1993, pp. 640-682. MR 94a:32038

Mathematical Institute of the Academy of Sciences, Žitná 25, 11567 Prague 1, Czech REPUBLIC

E-mail address: englis@math.cas.cz 